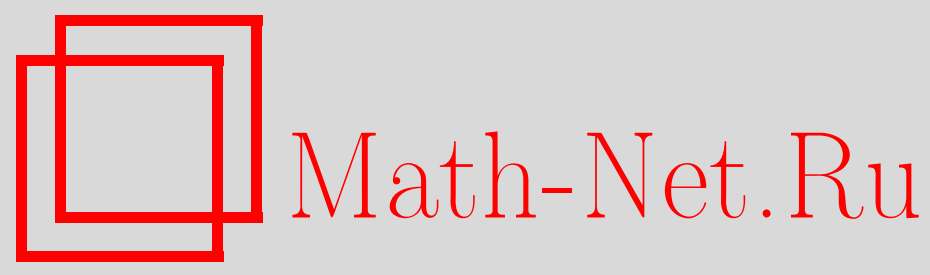

О. В. Бесов, Эквивалентные нормы в пространствах функций дробной гладкости на произвольной области, Матем. заметки, 2003, том 74, выпуск 3, 340-349

DOI: https://doi.org/10.4213/mzm267

Использование Общероссийского математического портала Math-Net.Ru подразумевает, что вы прочитали и согласны с пользовательским соглашением http://www . mathnet.ru/rus/agreement

Параметры загрузки:

IP: 54.157 .27 .8

26 апреля 2023 г., 15:32:56

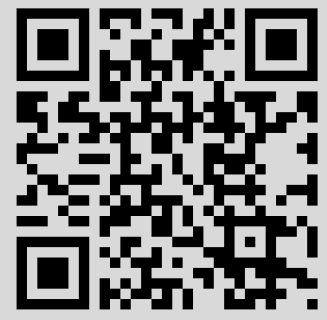




\section{ЭКВИВАЛЕНТНЫЕ НОРМЫ В ПРОСТРАНСТВАХ ФУНКЦИЙ ДРОБНОЙ ГЛАДКОСТИ НА ПРОИЗВОЛЬНОЙ ОБЛАСТИ}

О.В. Бесов

Здесь изучаются пространства $B_{p q}^{s}(G), L_{p q}^{s}(G)$ функций $f$ с положительньм показателем гладкости $s>0$, заданных на области $G \subset \mathbb{R}^{n}$. Нормы в этих пространствах определены с помощью интегральных норм разности функции $f$ порядка $m>s$, рассматриваемой как функция точки области и шага разности. Для произвольной области $G \subset \mathbb{R}^{n}$ устанавливается характеризация этих пространств в терминах локальных аппроксимаций функции многочленами степени $m-1$.

Библиография: 16 названий.

Пусть $\mathbb{N}$ - множество натуральных чисел, $n \in \mathbb{N}, n \geqslant 2, \mathbb{R}^{n}$ - евклидово пространство точек $x=\left(x_{1}, \ldots, x_{n}\right)=\sum_{1}^{n} x_{i} e^{i}, e^{i}$-орты стандартного базиса. Для $x, y \in \mathbb{R}^{n}$, $t>0, E \subset \mathbb{R}^{n}$ пусть $[x, y]-$ отрезок с концами в точках $x, y, y+t E=\{x: x=y+t z$, $z \in E\}$,

$$
B(x, t)=\{y:|y-x| \leqslant t\}=x+B(0, t), \quad B_{0}=B(0,1),
$$

$\chi$ - характеристическая функция шара $B_{0}$ или отрезка $[-1,1]$.

При $m \in \mathbb{N}$ положим

$$
\begin{gathered}
\Delta^{m}(y) f(x)=\sum_{j=0}^{m}(-1)^{m-j}\left(\begin{array}{c}
m \\
j
\end{array}\right) f(x+j y), \\
\Delta^{m}(y, E) f(x)= \begin{cases}\Delta^{m}(y) f(x) & \text { при } B(x, m|y|) \subset E, \\
0 & \text { при } B(x, m|y|) \not \subset E,\end{cases}
\end{gathered}
$$

при $t>0$

$$
\delta^{m}(t, E) f(x)= \begin{cases}\left|B_{0}\right|^{-1} \int_{|y|<1}\left|\Delta^{m}(t y) f(x)\right| d y & \text { при } B(x, m t) \subset E, \\ 0 & \text { при } B(x, m t) \not \subset E .\end{cases}
$$

Все встречающиеся множества измеримы по Лебегу, $|E|$ - мера Лебега множества $E \subset \mathbb{R}^{n}$. Все встречающиеся функции действительнозначны и локально суммируемы.

Работа выполнена при финансовой поддержке Российского фонда фундаментальных исследований, грант № 02-01-00602, программы "Ведущие научные шполы", № 00-15-96047, и INTAS, грант № 99-01080. 
Для $f: E \rightarrow \mathbb{R}, 1 \leqslant p \leqslant \infty$

$$
\|f\|_{p, E}=\left\|f \mid L_{p}(E)\right\|=\left(\int_{E}|f(x)|^{p} d x\right)^{\frac{1}{p}}, \quad\|f\|_{p}=\|f\|_{p, \mathbb{R}^{n}}
$$

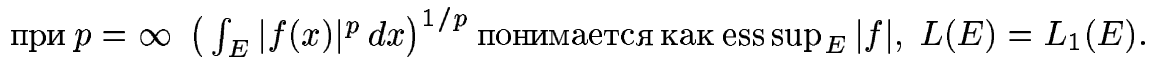

При $1<q<\infty$ через $L_{q}^{*}$ обозначается пространство измеримых на $(0,1)$ функций $g$ с нормой

$$
\left\|g \mid L_{q}^{*}\right\|=\left(\int_{0}^{1}|g(t)|^{q} \frac{d t}{t}\right)^{\frac{1}{q}}<\infty, \quad L_{\infty}^{*}=L_{\infty}((0,1)) .
$$

Через $G$ будем обозначать область в $\mathbb{R}^{n}$. При $\delta>0$ полагаем $G_{\delta}=\{x: x \in G$, $\operatorname{dist}(x, \partial G)>\delta\}$. При этом $\delta$ считаем столь малым, что $G_{\delta} \neq \varnothing$.

ОПРЕДЕЛЕНИЕ 1. Символом $B_{p q, r}^{s(m)}(G)$ при $1 \leqslant p, q, r \leqslant \infty, 0<s<m, m \in \mathbb{N}$, будем обозначать банахово пространство функций $f: G \rightarrow \mathbb{R}^{1}$ с нормой

$$
\left\|f\left|B_{p q, r}^{s(m)}(G)\|=\| f\right| L_{r}\left(G_{\delta}\right)\right\|+\left\|f \mid b_{p q}^{s(m)}(G)\right\|
$$

где

$$
\left\|f \mid b_{p q}^{s(m)}(G)\right\|=\left(\int_{m|h|<1}\left(\frac{\left\|\Delta^{m}(h, G) f\right\|_{p}}{|h|^{s}}\right)^{q} \frac{d h}{|h|^{n}}\right)^{\frac{1}{q}} .
$$

ОПРЕДЕЛЕНИЕ 2. Символом $L_{p q, r}^{s(m)}(G)$ при $1<p, q \leqslant \infty, 0<s<m, m \in \mathbb{N}$, будем обозначать банахово пространство функций $f: G \rightarrow \mathbb{R}^{1}$ с нормой

$$
\left\|f\left|L_{p q, r}^{s(m)}(G)\|=\| f\right| L_{r}\left(G_{\delta}\right)\right\|+\left\|f \mid l_{p q}^{s(m)}(G)\right\|,
$$

где

$$
\left\|f \mid l_{p q}^{s(m)}(G)\right\|=\left\|\left(\int_{0}^{\frac{1}{m}}\left(\frac{\delta^{m}(t, G) f}{t^{s}}\right)^{q} \frac{d t}{t}\right)^{\frac{1}{q}}\right\|_{p} .
$$

При $r=p$ вместо $B_{p q, r}^{s(m)}(G), L_{p q, r}^{s(m)}(G)$ и т.п. будем писать $B_{p q}^{s(m)}(G), L_{p q}^{s(m)}(G)$ и т.п. При $m-1 \leqslant s<m$ верхний индекс $(m)$ в обозначениях пространств будем опускать.

Пространства $B_{p q}^{s}\left(\mathbb{R}^{n}\right), L_{p q}^{s}\left(\mathbb{R}^{n}\right)\left(=F_{p q}^{s}\left(\mathbb{R}^{n}\right)\right)$ изучены достаточно подробно (см. [1][3]). Характеризация пространств Лизоркина-Трибеля $L_{p q}^{s}$ на $\mathbb{R}^{n}$ и на областях $G \subset$ $\mathbb{R}^{n}$ с достаточно гладкой границей в терминах шаровых средних от абсолютных величин разностей была предложена Стрихартцем [4] при $q=2$ (пространства бесселевых потенциалов) и в общем случае Лизоркиным [5]. Другие характеризации пространств $B_{p q}^{s}(G), L_{p q}^{s}(G)$ на области $G$ с регулярной границей можно найти в [3], [6].

Введем в рассмотрение также пространства функций, характеризуемых определенными интегральными оценками для их локальных приближений алгебраическими многочленами. Примем некоторые обозначения:

$$
\tau_{a} f(x)=f(x+a), \quad a \in \mathbb{R}^{n}, \quad \sigma_{t} f(x)=f(t x), \quad t>0,
$$


$\mathscr{P}_{m-1}$ - подпространство пространства $L\left(B_{0}\right)$, состоящее из всех многочленов вида $\sum_{|\alpha| \leqslant m-1} c_{\alpha} x^{\alpha}$ (степени не выше $m-1$ ),

$$
\begin{gathered}
\pi=\pi^{(m-1)}-\text { некоторый проектор } L\left(B_{0}\right) \text { на } \mathscr{P}_{m-1}, \\
\pi_{a, t}=\tau_{a}^{-1} \circ \sigma_{t}^{-1} \circ \pi \circ \sigma_{t} \circ \tau_{a}, \\
D_{m-1}(t, E) f(x)=D_{m-1}(t, B(x, t) \cap E) f(x) \\
= \begin{cases}t^{-n}\left\|f-\pi_{x, t} f \mid L(B(x, t))\right\| \text { при } B(x, t) \subset E, \\
0 & \text { при } B(x, t) \not \subset E .\end{cases}
\end{gathered}
$$

ЗАмЕчание 1 [7]. Функционалы $D_{m-1}(t, G) f$, образованные с помощью двух разных проекторов $\pi$ и $\hat{\pi}$, эквивалентны с постоянными, не зависящими от $f, x, t$. В самом деле,

$$
\begin{aligned}
\left\|f-\hat{\pi}_{x, t} f \mid L(B(x, t))\right\| & =\left\|f-\pi_{x, t} f-\hat{\pi}_{x, t}\left(f-\pi_{x, t} f\right) \mid L(B(x, t))\right\| \\
& \leqslant(1+\|\hat{\pi}\|)\left\|f-\pi_{x, t} f \mid L(B(x, t))\right\| .
\end{aligned}
$$

Из аналогичных оценок ясно также, что $D_{m-1}(t, G) f$ останется эквивалентньм после замены в нем приближения функции с помощью проекционного многочлена на ее наилучшее приближение.

ОПРЕДЕЛЕНИЕ 3 . При $1 \leqslant p, q, r \leqslant \infty, 0<s<m, m \in \mathbb{N}$, обозначим через $\widetilde{B}_{p q, r}^{s(m)}(G), \widetilde{L}_{p q, r}^{s(m)}(G)$ пространства функций $f: G \rightarrow \mathbb{R}^{1}$ с конечными нормами соответственно

$$
\begin{aligned}
\left\|f \mid \widetilde{B}_{p q, r}^{s(m)}(G)\right\| & =\left\|f\left|L_{r}\left(G_{\delta}\right)\|+\| f\right| \tilde{b}_{p q}^{s(m)}(G)\right\| \\
\left\|f \mid \widetilde{L}_{p q, r}^{s(m)}(G)\right\| & =\left\|f\left|L_{r}\left(G_{\delta}\right)\|+\| f\right| \tilde{l}_{p q}^{s(m)}(G)\right\|
\end{aligned}
$$

где

$$
\begin{aligned}
& \left\|f \mid \tilde{b}_{p q}^{s(m)}(G)\right\|=\left(\int_{0}^{1}\left\|D_{m-1}(t, G) f\right\|_{p}^{q} t^{-s q} \frac{d t}{t}\right)^{1 / q} \\
& \left\|f \mid \tilde{l}_{p q}^{s(m)}(G)\right\|=\left\|\left(\int_{0}^{1}\left(D_{m-1}(t, G) f\right)^{q} t^{-s q} \frac{d t}{t}\right)^{1 / q}\right\|_{p}
\end{aligned}
$$

Через $b_{p q}^{s(m)}(G), l_{p q}^{s(m)}(G), \tilde{b}_{p q}^{s(m)}(G), \tilde{l}_{p q}^{s(m)}(G)$ будем обозначать полунормированные пространства с полунормами, введенными в определениях 1-3.

Пространства функций, характеризуемых локальными приближениями многочленами, изучались во многих работах, см., например, [2], [4], [7]-[9]. Ближайшими к представленным здесь результатам о совпадении $B_{p q}^{s}(G)=\widetilde{B}_{p q}^{s}(G), L_{p q}^{s}(G)=\widetilde{L}_{p q}^{s}(G)$ являются результаты Аджиева [7], установившего их для областей $G$ с регулярной границей. Этим он дополнил для пространств $B_{p q}^{s}(G)$ предшествующие результаты Ильина (см. [2]) и распространил на области более общего вида результаты Трибеля [8] для областей с бесконечно гладкой границей. Отметим еще результаты Девора и Шарпли [10], 
исследовавших для регулярной области $G \subset \mathbb{R}^{n}$ шкалу пространств $C_{p}^{s}(G)=\widetilde{L}_{p, \infty}^{s}(G)$. Другие справки исторического характера можно найти в [7], [3], [6].

Из относящихся к произвольной области $G \subset \mathbb{R}^{n}$ отметим результат Кальдерона [11] о характеризации пространства Соболева $W_{p}^{l}(G)$ в терминах локальных аппроксимаций.

При доказательстве эквивалентности полунорм в пространстве $l_{p q}^{s(m)}(G)$ существенно будет использована

Tеорема I [12]-[14]; [15, Т. 2, С. 335], [3, п. 2.2.2]. Пусть $B_{1}, B_{2}-$ рефлексивные банаховы пространства и оператор $A$ определен на функииях $\bar{f} \in L_{p}\left(\mathbb{R}^{n}, B_{1}\right), p \in$ $(1, \infty)$, формулой

$$
(A \bar{f})(x)=\int_{\mathbb{R}^{n}} K(x-y) \bar{f}(y) d y
$$

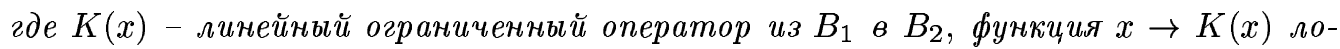
кально суммируема на $\mathbb{R}^{n}$. Пусть при некоторых $p_{0} \in(1, \infty), R, M>0$

1) $\quad\left\|A \bar{f}\left|L_{p_{0}}\left(\mathbb{R}^{n}, B_{2}\right)\|\leqslant M\| \bar{f}\right| L_{p_{0}}\left(\mathbb{R}^{n}, B_{1}\right)\right\| ;$

2) $\int_{|x|>R|y|}\left\|K(x)-K(x-y) \mid B_{1} \rightarrow B_{2}\right\| d x \leqslant M \quad \forall y \in \mathbb{R}^{n}$.

Тогда для всех $p \in(1, \infty)$

$$
\left\|A \bar{f}\left|L_{p}\left(\mathbb{R}^{n}, B_{2}\right)\left\|\leqslant C_{p} M\right\| \bar{f}\right| L_{p}\left(\mathbb{R}^{n}, B_{1}\right)\right\|,
$$

где $C_{p}$ не зависит от $\bar{f}, M$.

ТЕорема 1. Для произвольной области $G \subset \mathbb{R}^{n}$ при $0<s<m, 1 \leqslant p, q \leqslant \infty$ полунормы $\left\|f\left|b_{p q}^{s(m)}(G)\|u\| f\right| \tilde{b}_{p q}^{s(m)}(G)\right\|$ әквивалентны.

ТЕОрема 2. Для произвольной области $G \subset \mathbb{R}^{n}$ при $0<s<m$ и при $1<p, q<\infty$ или $1<p \leqslant q=\infty$ полунормы $\left\|f\left|l_{p q}^{s(m)}(G)\|u\| f\right| \tilde{l}_{p q}^{s(m)}(G)\right\|$ әквивалентны.

ДокАЗАТЕЛЬСтво ТЕОрем 1,2 . Оценим сначала $D_{m-1}\left(1, B_{0}\right) f(0)$ через интеграл от $\delta^{m}\left(t, B_{0}\right) f$. Воспользуемся для этого проекционньм представлением из [16]:

$$
f(x)=P_{m-1}(x)+\int_{0}^{1} \iint R(x, y, z) \Delta^{m}(v y) f(x-v x+v y+v z) d y d z \frac{d v}{v},
$$

где $P_{m-1}(x)$ - (проекционный) многочлен (степени $\left.m-1\right)$, построенный по значениям функции $f$ из шара $B_{0}, R(x, y, z)$ - многочлен степени $m$ по $x$ с коэффициентами из $C_{0}^{\infty}$ по $y$ и по $z$, сосредоточенньми в $\{(y, z):|y|<\varepsilon,|z|<\varepsilon\}$, где число $\varepsilon>0$ можно взять произвольно мальм. Поэтому в (5) при $x \in B_{0}$ вместо $\Delta^{m}(v y) f$ можно написать $\Delta^{m}\left(v y, B_{0}\right) f$. Из (5) видно, что

$$
\begin{aligned}
D_{m-1}\left(1, B_{0}\right) f(0) & =\left\|f-\pi f\left|L_{1}\left(B_{0}\right)\|\leqslant\| f-P_{m-1}-\pi\left(f-P_{m-1}\right)\right| L_{1}\left(B_{0}\right)\right\| \\
& \leqslant C_{1}\left\|f-P_{m-1} \mid L_{1}\left(B_{0}\right)\right\| \\
& \leqslant C_{2}\left(\int_{0}^{\frac{1}{2}}+\int_{\frac{1}{2}}^{1}\right) \int_{|y|<1}\left\|\Delta^{m}\left(v y, B_{0}\right) f\right\|_{1} d y \frac{d v}{v} .
\end{aligned}
$$


Для получения последней оценки при $v \leqslant 1 / 2$ сначала интегрирование было проведено по $x$, а затем по $z ;$ при $v>1 / 2$ - в противоположном порядке.

Из (6) имеем

$$
D_{m-1}\left(1, B_{0}\right) f(0) \leqslant C_{3} \int_{0}^{1}\left\|\delta^{m}\left(v, B_{0}\right) f\right\|_{1} \frac{d v}{v} .
$$

Отсюда с помощью сдвигов и растяжений получаем, что при $t>0, x \in G_{t}$

$$
\begin{aligned}
D_{m-1}(t, G) f(x) & =D_{m-1}(t, B(x, t)) f(x)=D_{m-1}(t, B(0, t))\left(\tau_{x} f\right)(0) \\
& =D_{m-1}\left(1, B_{0}\right)\left(\sigma_{t} \tau_{x} f\right)(0) \leqslant C_{3} \int_{0}^{1}\left\|\delta^{m}(t v, B(x, t)) f\right\|_{1} \frac{d v}{v} \\
& =C_{3} t^{-n} \int_{0}^{1}\left\|\delta^{m}(t v, B(x, t)) f\right\|_{1} \frac{d v}{v} \\
& =C_{3} t^{-n} \int_{0}^{t}\left\|\delta^{m}(v, B(x, t)) f\right\|_{1} \frac{d v}{v} .
\end{aligned}
$$

Продолжая оценку (7), имеем

$$
\begin{aligned}
D_{m-1}(t, G) f(x) & \leqslant C_{4} t^{-n} \int_{0}^{t} \int_{|y|<1}\left\|\Delta^{m}(v y, B(x, t)) f\right\|_{1} d y \frac{d v}{v} \\
& =C_{4} t^{-n} \int_{0}^{t} \int_{|y|<v}\left\|\Delta^{m}(y, B(x, t)) f\right\|_{1} d y \frac{d v}{v^{n+1}} \\
& \leqslant C_{5} t^{-n} \int_{|y|<t}\left\|\Delta^{m}(y, B(x, t)) f\right\|_{1} \frac{d y}{|y|^{n}} .
\end{aligned}
$$

С помощью неравенства Гёльдера имеем

$$
\left\|\Delta^{m}(y, B(x, t)) f\right\|_{1} \leqslant C_{6}\left\|\Delta^{m}(y, B(x, t)) f\right\|_{p} t^{n / p^{\prime}} .
$$

Из (8) с помощью неравенства Минковского и (9) получаем оценку

$$
\begin{aligned}
& \left\|D_{m-1}(t, G) f\right\|_{p} \\
& \quad \leqslant C_{7} t^{-n} \int_{|y|<t}\left\|\Delta^{m}(y, B(x, t)) f\right\|_{p} t^{\frac{n}{p^{\prime}}}\left[\int_{G}\left\|\Delta^{m}(y, B(x, t)) f\right\|_{p}^{p} d x\right]^{\frac{1}{p}} \frac{d y}{|y|^{n}} \\
& \quad \leqslant C_{8} \int_{|y|<t}\left\|\Delta^{m}(y, G) f\right\|_{p} \frac{d y}{|y|^{n}} .
\end{aligned}
$$

Применяя неравенство Гёльдера, из (10) при $0<\kappa<s, q<\infty$ получаем

$$
\begin{aligned}
& \left(\int_{0}^{1}\left\|D_{m-1}(t, G) f\right\|_{p}^{q} t^{-s q} \frac{d t}{t}\right)^{1 / q} \leqslant C_{8}\left(\int_{0}^{1}\left[\int_{|y|<t}\left\|\Delta^{m}(y, G) f\right\|_{p} \frac{d y}{|y|^{n}}\right]^{q} t^{-s q} \frac{d t}{t}\right)^{1 / q} \\
& \quad \leqslant C_{8}\left(\int_{0}^{1} \int_{|y|<t} \frac{\left\|\Delta^{m}(y, G) f\right\|_{p}^{q}}{|y|^{\kappa q}} \frac{d y}{|y|^{n}}\left[\int_{|y|<t}|y|^{\kappa q^{\prime}} \frac{d y}{|y|^{n}}\right]^{q / q^{\prime}} t^{-s q} \frac{d t}{t}\right)^{1 / q} \\
& \quad \leqslant C_{9}\left(\int_{0}^{1} \int_{|y|<t}\left\|\Delta^{m}(y, G) f\right\|_{p}^{q}|y|^{-\kappa q} \frac{d y}{|y|^{n}} t^{\kappa q-s q} \frac{d t}{t}\right)^{1 / q} \\
& \quad \leqslant C\left(\int_{|y|<1}\left\|\Delta^{m}(y, G) f\right\|_{p}^{q}|y|^{-s q} \frac{d y}{|y|^{n}}\right)^{1 / q} .
\end{aligned}
$$


При $q=\infty$ оценка (11) выводится аналогично.

Из (11) следует вложение

$$
b_{p q}^{s(m)}(G) \subset \tilde{b}_{p q}^{s(m)}(G)
$$

для произвольной области $G \subset \mathbb{R}^{n}$.

Получим теперь вложение

$$
l_{p q}^{s(m)}(G) \subset \tilde{l}_{p q}^{s(m)}(G) \quad \text { при } 1<p, q<\infty \text { и при } 1<p \leqslant q=\infty .
$$

Пусть сначала $1<p, q<\infty$. Пусть $\chi_{0} \in C_{0}^{1}\left(\mathbb{R}^{n}\right.$ ) (или $\chi_{0} \in C_{0}^{1}\left(\mathbb{R}^{1}\right)$ ), причем $\chi(x)$ $\leqslant \chi_{0}(x) \leqslant \chi(x / 2)$ при всех $x \in \mathbb{R}^{n}$ (соответственно при всех $x \in \mathbb{R}^{1}$ ).

Из оценки (7) следует, что

$$
t^{-s} D_{m-1}(t, G) f(x) \leqslant C \int\left(K_{0}(y)\left((\cdot)^{-s} \delta^{m}(\cdot, G) f(x+y)\right)\right)(t) d y,
$$

где оператор $K_{\varepsilon}(y): L_{q}^{*} \rightarrow L_{q}^{*}$ имеет вид

$$
\left(K_{\varepsilon}(y) g\right)(t)=t^{-n} \chi_{0}\left(\frac{\varepsilon}{|y|}\right) \chi_{0}\left(\frac{y}{t}\right) \int_{0}^{t} g(v)\left(\frac{v}{t}\right)^{s} \frac{d v}{v}, \quad \varepsilon \geqslant 0
$$

Убедимся, что условия теоремы Івыполнены для оператора $A$ с ядром $K=K_{\varepsilon}, \varepsilon>0$, $B_{1}=B_{2}=L_{q}^{*}, p_{0}=q, R=2$.

Для $\bar{f}(v, x), \bar{f} \in L_{q}\left(\mathbb{R}^{n}, L_{q}^{*}\right)$, имеем

$$
N^{q}=\left\|A \bar{f} \mid L_{q}\left(\mathbb{R}^{n}, L_{q}^{*}\right)\right\|^{q} \leqslant \iint_{0}^{1}\left[\int t^{-n} \chi_{0}\left(\frac{y}{t}\right) \int_{0}^{t}|\bar{f}(v, x+y)|\left(\frac{v}{t}\right)^{s} \frac{d v}{v} d y\right]^{q} \frac{d t}{t} d x
$$

Применяя неравенство Гёльдера по $v, y$, получаем

$$
N^{q} \leqslant C \iint_{0}^{1} \int t^{-n} \chi_{0}\left(\frac{y}{t}\right) \int_{0}^{t}|\bar{f}(v, x+y)|^{q}\left(\frac{v}{t}\right)^{s q} \frac{d v}{v} d y \frac{d t}{t} d x .
$$

Интегрируя последовательно по $x, y, t$, получаем оценку

$$
N^{q} \leqslant C_{1} \int_{0}^{1}\left\|\bar{f}(v, \cdot)\left|L_{q}\left(\mathbb{R}^{n}\right)\left\|^{q} \frac{d v}{v}=C_{1}\right\| \bar{f}\right| L_{q}\left(\mathbb{R}^{n}, L_{q}^{*}\right)\right\|^{q} .
$$

Этим условие 1) теоремы I проверено.

Проверим условие 2). Заметим, что при $\varepsilon>0,0<t \leqslant 1$

$$
\begin{aligned}
\Delta(t, x, y) & =\chi_{0}\left(\frac{\varepsilon}{|x|}\right) \chi_{0}\left(\frac{x}{t}\right)-\chi_{0}\left(\frac{\varepsilon}{|x-y|}\right) \chi_{0}\left(\frac{x-y}{t}\right) \\
& =\left[\chi_{0}\left(\frac{\varepsilon}{|x|}\right)-\chi_{0}\left(\frac{\varepsilon}{|x-y|}\right)\right] \chi_{0}\left(\frac{x}{t}\right)+\chi_{0}\left(\frac{\varepsilon}{|x-y|}\right)\left[\chi_{0}\left(\frac{x}{t}\right)-\chi_{0}\left(\frac{x-y}{t}\right)\right],
\end{aligned}
$$


откуда с помощью теоремы Лагранжа о конечных приращениях получаем

$$
\begin{aligned}
|\Delta(t, x, y)| \leqslant & \chi_{0}\left(\frac{x}{t}\right) \max _{a \in\left[\frac{\varepsilon}{|x|}, \frac{\varepsilon}{|x-y|}\right]}\left|\chi_{0}^{\prime}(a)\right| \frac{\varepsilon|| x-y|-| x||}{|x||x-y|} \\
& +\chi_{0}\left(\frac{\varepsilon}{|x-y|}\right) \max _{z \in[x, x-y]}\left|\operatorname{grad} \chi_{0}\left(\frac{z}{t}\right)\right| \frac{|y|}{t} .
\end{aligned}
$$

Отсюда

$$
\sup _{0<t \leqslant 1}\left|\Delta(t, x, y) t^{-n}\right| \leqslant C_{1} \frac{|y|}{|x|^{n+1}} .
$$

Пусть $g \in L_{q}^{*}$. Тогда с помощью неравенства Минковского имеем

$$
\begin{aligned}
\|\left(K_{\varepsilon}(x)-\left(K_{\varepsilon}(x-y)\right) g \mid L_{q}^{*} \|\right. & \leqslant\left\{\int_{0}^{1}\left[|\Delta(t, x, y)| t^{-n} \int_{0}^{t}|g(v)|\left(\frac{v}{t}\right)^{s} \frac{d v}{v}\right]^{q} \frac{d t}{t}\right\}^{1 / q} \\
& \leqslant C_{1} \frac{|y|}{|x|^{n+1}}\left\{\int_{0}^{1}\left[\int_{0}^{1}|g(t u)| u^{s} \frac{d u}{u}\right]^{q} \frac{d t}{t}\right\}^{1 / q} \\
& \leqslant C_{1} \frac{|y|}{|x|^{n+1}} \int_{0}^{1}\left\{\int_{0}^{1}|g(t u)|^{q} \frac{d t}{t}\right\}^{1 / q} u^{s} \frac{d u}{u} \\
& \leqslant C_{2} \frac{|y|}{|x|^{n+1}}\left\|g \mid L_{q}^{*}\right\| .
\end{aligned}
$$

Теперь для проверки условия 2) теоремы I остается заметить, что

$$
\int_{|x|>2|y|} \frac{|y|}{|x|^{n+1}} d x \leqslant C \quad \forall y \neq 0 .
$$

Из приведенных выкладок видно, что условия 1), 2) для оператора $A$ с ядром $K_{\varepsilon}$ выполнены с постоянньми, не зависящими от $\varepsilon>0$. В силу этого с помощью предельного перехода при $\varepsilon \rightarrow 0$ под знаком интеграла Лебега заключаем, что будет ограниченньм в $L_{p}\left(\mathbb{R}^{n}, L_{q}^{*}\right)$ оператор, стоящий в правой части (14). Этим вложение (13) при $1<p, q<\infty$ установлено.

Установим вложение (13) в случае $1<p \leqslant q=\infty$. В силу (7) имеем

$$
\begin{aligned}
& \sup _{0<t<1} t^{-s} D_{m-1}(t, G) f(x) \\
& \quad \leqslant C \sup _{0<t<1} t^{-s-n} \int_{0}^{t} \int_{|y|<t} v^{s} \sup _{0<w \leqslant 1} w^{-s} \delta^{m}(w, G) f(x+y) d y \frac{d v}{v} \\
& \quad \leqslant C_{1} \sup _{0<t<1} t^{-n} \int_{|y|<t} \sup _{0<v \leqslant 1} v^{-s} \delta^{m}(v, G) f(x+y) d y,
\end{aligned}
$$

откуда переходом к $L_{p}$-норме в силу ограниченности оператора максимальной функции получаем желаемую оценку. Этим вложение (13) установлено.

Для получения вложений, противоположных вложениям (12), (13), также воспользуемся проекционным представлением (5). В правой части (5) вместо $f$ можно написать 
произвольный многочлен $Q_{m-1}$ степени не выше $m-1$ с коэффициентами, зависящими от $x$ и $v$. Оценивая разность $\Delta^{m}(h) f(0)$ как разность от правой части (5) через сумму модулей значений правой части при сдвинутых на $k h$ аргументах, имеем

$$
\begin{aligned}
& \left|\Delta^{m}(h) f(0)\right| \\
& \quad \leqslant C \sum_{k=0}^{m} \int_{0}^{1} \iint \chi\left(\frac{y}{\varepsilon}\right) \chi\left(\frac{z}{\varepsilon}\right) \sum_{j=1}^{m+1}\left|\left(f-Q_{m-1}\right)(k h(1-v)+j v y+v z)\right| d y d z \frac{d v}{v} .
\end{aligned}
$$

Здесь $m|h| \leqslant 1$, коэффициенты $Q_{m-1}$ зависят от $h, k, v, f: B_{0} \rightarrow \mathbb{R}$. Выбирая $Q_{m-1}$ надлежащим образом, имеем

$$
\left|\Delta^{m}(h) f(0)\right| \leqslant C \sum_{k=0}^{m} \int_{0}^{\frac{1}{2}} D_{m-1}\left(v, B_{0}\right) f(k h(1-v)) \frac{d v}{v}+C \int_{\frac{1}{2}}^{1} D_{m-1}\left(v, B_{0}\right) f(0) \frac{d v}{v} .
$$

Пусть $f: B(0, t) \rightarrow \mathbb{R}^{1}$. Применяя последнюю оценку к функции $f_{t}(x)=f(t x)$, получаем при $x=0, G=B(0, t)$ оценку

$$
\begin{aligned}
\left|\Delta^{m}(t h, G) f(x)\right| \leqslant & C \sum_{k=1}^{m} \int_{0}^{\frac{t}{2}} D_{m-1}(v, G) f\left(x+k t h\left(1-\frac{v}{t}\right)\right) \frac{d v}{v} \\
& +C \int_{0}^{t} D_{m-1}(v, G) f(x) \frac{d v}{v}
\end{aligned}
$$

где $m|h| \leqslant 1,0<t<\min \left\{1, \operatorname{dist}\left(x, \mathbb{R}^{n} \backslash G\right)\right\}$.

$\mathrm{C}$ помощью сдвига видим, что оценка (15) справедлива для произвольного $x \in \mathbb{R}^{n}$ при $G=B(x, t)$. Но тогда она верна в приведенном в $(15)$ виде. Считая в $(15) h=1 / m$ и положив $y=t h$, получим

$$
\begin{aligned}
\left|\Delta^{m}(y, G) f(x)\right| \leqslant & C \sum_{k=1}^{m} \int_{0}^{2 m|y|} D_{m-1}(v, G) f\left(x+k h\left(1-\frac{v}{t}\right)\right) \frac{d v}{v} \\
& +C \int_{0}^{2 m|y|} D_{m-1}(v, G) f(x) \frac{d v}{v}, \quad|y|>0
\end{aligned}
$$

(при $m|y| \geqslant \operatorname{dist}\left\{x, \mathbb{R}^{n} \backslash G\right\}$ левая часть равна нулю). Переходя к $L_{p}(G)$-нормам, получаем отсюда

$$
\left\|\Delta^{m}(y, G) f\right\|_{p} \leqslant C_{1} \int_{0}^{2 m|y|}\left\|D_{m-1}(v, G) f\right\|_{p} \frac{d v}{v} .
$$

Применяя неравенство Гёльдера, при $0<\sigma<s, 1 \leqslant q<\infty$ получаем

$$
\left\|\Delta^{m}(y, G) f\right\|_{p} \leqslant C_{2}|y|^{\sigma}\left(\int_{0}^{2 m|y|}\left(v^{-\sigma}\left\|D_{m-1}(v, G) f\right\|_{p}\right)^{q} \frac{d v}{v}\right)^{1 / q}
$$

откуда

$$
\left(\int_{m|y|<1}\left\|\Delta^{m}(y, G) f\right\|_{p}^{q} \frac{d y}{|y|^{s q+n}}\right)^{1 / q} \leqslant C_{3}\left(\int_{0}^{1}\left\|D_{m-1}(v, G) f\right\|_{p}^{q} \frac{d v}{v^{s q+1}}\right)^{1 / q}
$$


Это неравенство устанавливается без применения неравенства Гёльдера и при $q=\infty$.

Из (16) следует, что

$$
\tilde{b}_{p q}^{s(m)}(G) \subset b_{p q}^{s(m)}(G)
$$

Установим вложение

$$
\tilde{l}_{p q}^{s(m)}(G) \subset l_{p q}^{s(m)}(G)
$$

при $1<p, q<\infty$ и при $1<p \leqslant q=\infty$.

Интегрируя (15) по $h$ при $m|h|<1$, получаем

$$
\begin{aligned}
\delta^{m}(t, G) f(x) \leqslant & C \iint_{0}^{\frac{t}{2}} D_{m-1}(v, G) f(x+y) t^{-n} \chi_{0}\left(\frac{y}{t}\right) \frac{d v}{v} d y \\
& +C \int_{0}^{t} D_{m-1}(v, G) f(x) \frac{d v}{v}
\end{aligned}
$$

Отсюда в обозначениях $g(v, x)=v^{-s} D_{m-1}(v, G) f(x)$ имеем

$$
t^{-s} \delta^{m}(t, G) f(x) \leqslant C A g(t, x)+C B g(t, x),
$$

где

$$
\begin{gathered}
A g(t, x)=\int(K(y) g(\cdot, x+y))(t) d y \\
(K(y) \phi)(t)=t^{-s-n} \chi_{0}\left(\frac{y}{t}\right) \int_{0}^{t} v^{s} \phi(v) \frac{d v}{v}, \quad \phi \in L_{q}^{*} \\
(B g)(t, x)=t^{-s} \int_{0}^{t} v^{s} g(v, x) \frac{d v}{v}=\int_{0}^{1} u^{s} g(t u, x) \frac{d u}{u} .
\end{gathered}
$$

Ограниченность оператора $A: L_{p}\left(L_{q}^{*}\right) \rightarrow L_{p}\left(L_{q}^{*}\right)$ установлена при доказательстве (13).

Для доказательства ограниченности оператора $B: L_{p}\left(L_{q}^{*}\right) \rightarrow L_{p}\left(L_{q}^{*}\right)$ получаем сначала с помощью неравенства Гёльдера оценку $(0<\varepsilon<s)$

$$
(B g)(t, x) \leqslant C t^{-\varepsilon}\left(\int_{0}^{t} v^{\varepsilon q} g(v, x)^{q} \frac{d v}{v}\right)^{1 / q}
$$

откуда

$$
\begin{aligned}
\left\|(B g)(\cdot, x) \mid L_{q}^{*}\right\|^{q} & \leqslant C^{q} \int_{0}^{1} t^{-\varepsilon q} \int_{0}^{t} v^{\varepsilon q} g(v, x)^{q} \frac{d v}{v} \frac{d t}{t} \\
& \leqslant C_{1}^{q} \int_{0}^{1} g(v, x)^{q} \frac{d v}{v}=C_{1}^{q}\left\|g(\cdot, x) \mid L_{q}^{*}\right\|^{q} .
\end{aligned}
$$

Теперь остается лишь перейти к $L_{p}(G)$-нормам в последнем неравенстве. Из (19) и установленной ограниченности операторов $A, B$ следует (18) при $1<p, q<\infty$.

В случае $1<p \leqslant q=\infty$ доказательство (18) элементарно следует из оценки (19) и ограниченности оператора максимальной функции в $L_{p}$ при $1<p \leqslant \infty$. Тем самым теоремы 1, 2 установлены. 


\section{СПИСОК ЦИТИРОВАННОЙ ЛИТЕРАТУРЫ}

[1] Никольский С. М. Приближение функций многих переменных и теоремы вложения. М.: Наука, 1977.

[2] Бесов О. В., Ильин В.П., Никольский С. М. Интегральные представления функций и теоремы вложения. М.: Наука, 1996.

[3] Трибель Х. Теория интерполяции, функциональные пространства, дифференциальные операторы. М.: Мир, 1980.

[4] Strichartz P.S. Multipliers on fractional Sobolev spaces // J. Math. Mech. 1967. V. 16. № 9. P. 1031-1060.

[5] Лизоркин П.И. Операторы, связанные с дробньм дифференцированием, и классы дифференцируемых функций // Тр. МИАН. 1972. Т. 117. С. 212-243.

[6] Трибель Х. Теория функциональных пространств. М.: Мир, 1986.

[7] Аджиев С. С. Характеризация функциональных пространств $B_{p, q}^{s}(G), L_{p, q}^{s}(G), W_{p}^{s}(G)$ и вложения в $B M O(G) / /$ Тр. МИАН. 1997. Т. 214. С. 7-24.

[8] Triebel H. Local approximation spaces // Z. Anal. und Anwend. 1989. V. 88. № 3. P. 261-288.

[9] Брудный Ю.А. Пространства, определяемые с помощью локальных приближений // Tp. MMO. 1971. T. 24. C. 71-132.

[10] DeVore R. A., Sharpley R.S. Maximal function measuring smoothness // Mem. Amer. Math. Soc. 1984. V. 293.

[11] Calderón A.P. Estimates for singular integral operators in terms of maximal functions // Stud. Math. 1972. V. 44. P. 563-682.

[12] Schwartz J. A remark on inequalities of Calderón-Zygmund type for vector-valued functions // Comm. Pure and Appl. Math. 1961. V. 14. № 4. P. 785-799.

[13] Бендек А., Кальдерон А. П., Панцоне Р. Операторы свертки на функциях со значениями в банаховом пространстве // Математика. 1963. Т. 7. № 5. С. 121-131.

[14] Krée P. Propriétés de continuité dans $L^{p}$ de certains noyaux // Boll. Unione Mat. Ital. 1967. V. 22. № 3. P. 330-334.

[15] Данфорд Н., Шварц Дж. Т. Линейные операторы. Спектральная теория. М.: Мир, 1966.

[16] Бесов О.В., Ильин В.П. Проекционные представления функций через разности // Тр. МИАН. 1979. Т. 150. С. 3-10. 